\title{
PRECISION TIMING OF PSR J0437-4715: AN ACCURATE PULSAR DISTANCE, A HIGH PULSAR MASS, AND A LIMIT ON THE VARIATION OF NEWTON'S GRAVITATIONAL CONSTANT
}

\author{
J. P. W. Verbiest, ${ }^{1,2}$ M. Bailes, ${ }^{1}$ W. van Straten, ${ }^{1}$ G. B. Hobbs,${ }^{2}$ R. T. Edwards, ${ }^{2}$ R. N. Manchester ${ }^{2}$ \\ N. D. R. Bhat, ${ }^{1}$ J. M. Sarkissian, ${ }^{2}$ B. A. Jacoby, ${ }^{3}$ and S. R. KulKarni ${ }^{4}$ \\ Received 2007 October 4; accepted 2008 January 16
}

\begin{abstract}
Analysis of 10 years of high-precision timing data on the millisecond pulsar PSR J0437-4715 has resulted in a model-independent kinematic distance based on an apparent orbital period derivative, $\dot{P}_{b}$, determined at the $1.5 \%$ level of precision $\left(D_{k}=157.0 \pm 2.4 \mathrm{pc}\right)$, making it one of the most accurate stellar distance estimates published to date. The discrepancy between this measurement and a previously published parallax distance estimate is attributed to errors in the DE200 solar system ephemerides. The precise measurement of $\dot{P}_{b}$ allows a limit on the variation of Newton's gravitational constant, $|\dot{G} / G| \leq 23 \times 10^{-12} \mathrm{yr}^{-1}$. We also constrain any anomalous acceleration along the line of sight to the pulsar to $\left|a_{\odot} / c\right| \leq 1.5 \times 10^{-18} \mathrm{~s}^{-1}$ at $95 \%$ confidence, and derive a pulsar mass, $m_{\mathrm{psr}}=1.76 \pm$ $0.20 M_{\odot}$, one of the highest estimates so far obtained.
\end{abstract}

Subject headings: pulsars: individual (PSR J0437-4715) — stars: distances — stars: neutron

\section{INTRODUCTION}

In 1993, Johnston et al. reported the discovery of PSR J04374715 , the nearest and brightest millisecond pulsar known. Within a year, the white dwarf companion and pulsar wind bow shock were observed (Bell et al. 1993) and pulsed X-rays were detected (Becker \& Trümper 1993). The proper motion and an initial estimate of the parallax were later presented along with evidence for secular change in the inclination angle of the orbit due to proper motion (Sandhu et al. 1997). Using high time resolution instrumentation, the three-dimensional orbital geometry of the binary system was determined, enabling a new test of general relativity (GR; van Straten et al. 2001). Most recently, multifrequency observations were used to compute the dispersion measure structure function (You et al. 2007), quantifying the turbulent character of the interstellar medium toward this pulsar.

The high proper motion and proximity of PSR J0437-4715 led to the prediction (Bell \& Bailes 1996) that a distance measurement independent of parallax would be available within a decade, when the orbital period derivative $\left(\dot{P}_{b}\right)$ would be determined to high accuracy. Even if the predicted precision of $\sim 1 \%$ would not be achieved, such a measurement would be significant given the strong dependence of most methods of distance determination on relatively poorly constrained models and the typically large errors on parallax measurements. Even for nearby stars, both the Hubble Space Telescope and the Hipparcos satellite give typical distance errors of 3\% (Valls-Gabaud 2007) and so far only two distances beyond $100 \mathrm{pc}$ have been determined at $\sim 1 \%$ uncertainty (Torres et al. 2007). This kinematic distance is one of the few model-independent methods that does not rely on the motion of the Earth around the Sun.

As demonstrated by Damour \& Taylor (1991) $\dot{P}_{b}$ can also be used to constrain the variation of Newton's gravitational constant. The best such limit from pulsar timing to date (Taylor 1993

\footnotetext{
1 Centre for Astrophysics and Supercomputing, Swinburne University of Technology, P.O. Box 218 Hawthorn, VIC 3122, Australia.

2 Australia Telescope National Facility-CSIRO, P.O. Box 76, Epping, NSW 1710, Australia.

3 Naval Research Laboratory, 4555 Overlook Avenue SW, Washington, DC 20375.

4 Robinson Laboratory, California Institute of Technology, Mail Code 105-24, Caltech Optical Observatories, Pasadena, CA 91125.
}

$|\dot{G} / G|=(4 \pm 5) \times 10^{-12} \mathrm{yr}^{-1}$ from PSR B1913+16) is compromised due to the poorly constrained equation of state for the neutron star companion (Nordtvedt 1990). The slightly weaker but more reliable limit of $|\dot{G} / G|=(-9 \pm 18) \times 10^{-12} \mathrm{yr}^{-1}$ (Kaspi et al. 1994 from PSR B1855+09, which has a white dwarf companion) should therefore be considered instead. A more stringent limit can be obtained from the Splaver et al. (2005) timing of PSR J1713+0747: $|\dot{G} / G|=(2.5 \pm 3.3) \times 10^{-12} \mathrm{yr}^{-1}$ (at $95 \%$ certainty). This limit is, however, based on the formal errors of $\dot{P}_{b}, P_{b}$, and parallax, which are easily underestimated by standard methodologies, as we shall demonstrate later. Because of this we believe the Splaver et al. (2005) limit is probably underestimated, but still of relevance. However, none of these limits are as strong as that put by lunar laser ranging (LLR; Williams et al. 2004): $\dot{G} / G=(4 \pm 9) \times 10^{-13} \mathrm{yr}^{-1}$. Besides limiting alternative theories of gravity, bounds on $\dot{G}$ can also be used to constrain variations of the astronomical unit (AU). Current planetary radar experiments (Krasinsky \& Brumberg 2004) have measured a significant linear increase of $d \mathrm{AU} / d t=0.15 \pm 0.04 \mathrm{~m} \mathrm{yr}^{-1}$, which may imply $\dot{G} / G=(-10 \pm 3) \times 10^{-13} \mathrm{yr}^{-1}$, just beyond the sensitivity of the limits listed above.

As mentioned before, the equation of state for dense neutron star matter is very poorly constrained. Pulsar mass determinations can probe the range of permissible pulsar masses and thereby limit possible equations of state (Lattimer \& Prakash 2007). Presently, only the pulsars NGC 6440B, Terzan 5 I and Terzan 5 J have predicted masses higher than the typical value of $1.4 M_{\odot}$ (Freire et al. 2008; Ransom et al. 2005); however, as discussed in more detail in $\S 5$, such predictions do not represent objective mass estimates.

The remainder of this paper is structured as follows. Section 2 describes the observations, data analysis and general timing solution for PSR J0437-4715. Section 3 describes how the measurement of $\dot{P}_{b}$ leads to a new and highly precise distance. In $\S 4$ this measurement is combined with the parallax distance to derive limits on $\dot{G}$ and the solar system acceleration. Section 5 presents the newly revised pulsar mass and our conclusions are summarized in $\S 6$.

\section{OBSERVATIONS AND DATA REDUCTION}

Observations of PSR J0437-4715 were made over a time span of 10 years (see Fig. 1), using the Parkes $64 \mathrm{~m}$ radio telescope, 


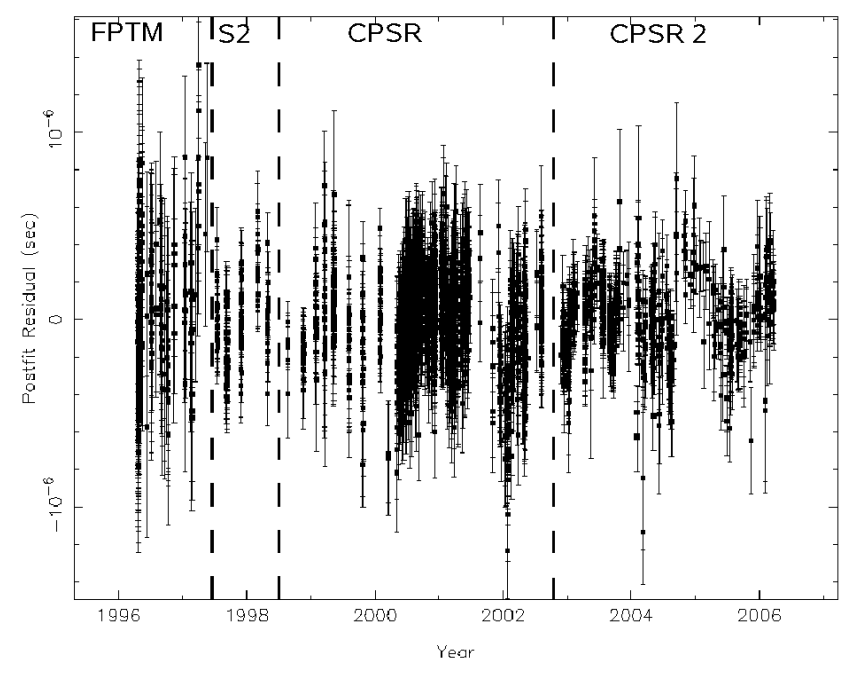

FIG. 1.-Combined $20 \mathrm{~cm}$ postfit timing residuals for new and archival PSR J0437-4715 timing data. Vertical dashed lines separate the different instruments.

two $20 \mathrm{~cm}$ receiving systems (the central beam of the Parkes multibeam receiver [Staveley-Smith et al. 1996] and the $\mathrm{H}-\mathrm{OH}$ receiver), and four generations of digital instrumentation (see Table 1): the Fast Pulsar Timing Machine (FPTM), the S2 VLBI recorder, and the Caltech-Parkes-Swinburne Recorders (CPSR and CPSR2). The FPTM is an autocorrelation spectrometer, whereas the three other instruments are baseband data recording and processing systems that employ phase-coherent dispersion removal.

\subsection{Arrival Time Estimation}

For the FPTM, S2, and CPSR back ends, the uncalibrated polarization data were combined to form the polarimetric invariant interval (Britton 2000), and each observation was integrated in time and frequency before pulse arrival times were calculated through standard cross-correlation with an instrument-dependent template profile. For the CPSR2 data, the technique described by van Straten (2004) was used to calibrate 5 days of intensive PSR J0437-4715 observations made on 2003 July 19 to 21, 2003 August 29, and 2005 July 24. The calibrated data were integrated to form a polarimetric template profile with an integration length of approximately $40 \mathrm{hr}$ and frequency resolution of $500 \mathrm{kHz}$. This template profile and Matrix Template Matching (MTM; van Straten 2006) were used to calibrate the 3 years of CPSR2 data. An independent MTM fit was performed on each 5 minute integration, producing a unique solution in each frequency channel, as shown in Figure 2 of van Straten (2006). The calibrated data were then integrated in frequency to produce a single fullpolarization profile at each epoch. MTM was then used to derive time-of-arrival (TOA) estimates from each calibrated, five-minute integration. The application of MTM during the calibration and timing stages reduced the weighted rms of the CPSR2 postfit timing residuals by a factor of 2 . All the data reduction described above was performed using the PSRCHIVE software package (Hotan et al. 2004).

\subsection{Timing Analysis}

Most data were recorded at a wavelength of $20 \mathrm{~cm}$; however, in the final 3 years, simultaneous observations at 10 and $50 \mathrm{~cm}$ were used to measure temporal variations of the interstellar dispersion delay (corrections for these variations were implemented in a way similar to that of You et al. 2007). A linear trend of these delays was also obtained for the year of FPTM data, using data at slightly different frequencies close to $1400 \mathrm{MHz}$.

The arrival times were analyzed using the Tempo 2 pulsar timing software package (Hobbs et al. 2006; Edwards et al. 2006) and consistency with the earlier program, Tempo, was verified. The timing model (see Table 2) is based on the relativistic binary model first derived by Damour \& Deruelle (1986) and expanded to contain the geometric orbital terms described by Kopeikin $(1995,1996)$. The model is optimized through a standard weighted least-squares fit in which all parameters are allowed to vary, including the unknown time delays between data from different instruments, but excluding the mean value of dispersion measure, which is determined from the simultaneous CPSR2, $64 \mathrm{MHz}$ wide bands centered at 1341 and $1405 \mathrm{MHz}$.

A major difference between our implementation of solutions for the orbital angles $\Omega$ and $i$ and previous efforts (van Straten et al. 2001; Hotan et al. 2006) is that they were implemented as part of the standard fitting routine. This ensures that any covariances between these and other parameters (most importantly the periastron advance and companion mass; see Table 2 and $\S 5$ ) are properly accounted for, thereby yielding a more reliable measurement error. The previous works mentioned above derived these effects from an independent mapping of $\chi^{2}$ space, leaving the errors of other parameters unaffected.

As can be seen from Figure 1, there are significant lowfrequency structures present in the timing residual data. Since the standard least-squares fitting routine used in Tempo 2 does not account for the effect of such correlations on parameter estimation, we performed a Monte Carlo simulation where data sets with a postfit power spectrum statistically consistent with that of the PSR J0437-4715 data were used to determine the parameter estimations uncertainties in the presence of realistic low-frequency noise. These errors, as well as the factors by which the original errors were underestimated, are shown in Table 2. As an example, the distribution of derived pulsar masses from the Monte Carlo simulation is given in Figure 2. Because of the dispersion measure corrections implemented in the final 3 years of data, one can

TABLE 1

Characteristics of the Timing Data for the Four Instruments Used

\begin{tabular}{|c|c|c|c|c|c|c|c|}
\hline Backend & Date Range & References & $\begin{array}{l}\text { Bandwidth } \\
\text { (MHz) }\end{array}$ & $\begin{array}{l}\text { rms Residual } \\
\text { (ns) }\end{array}$ & $\begin{array}{l}\text { Observation Length } \\
\text { (min) }\end{array}$ & Number of TOAs & $\begin{array}{l}\text { TOA Error }{ }^{\mathrm{a}} \\
\quad(\mathrm{ns})\end{array}$ \\
\hline FPTM ..................... & 1996 Apr-1997 May & 1,2 & 256 & 368 & 10 & 207 & 500 \\
\hline CPSR ....................... & 1998 Aug-2002 Aug & 3 & 20 & 218 & 15 & 1782 & 250 \\
\hline CPSR2 ..................... & 2002 Nov-2006 Mar & 4 & $2 \times 64^{\mathrm{b}}$ & 164 & 60 & 741 & 150 \\
\hline
\end{tabular}

\footnotetext{
${ }^{\text {a }}$ Displayed are typical values only.

b CPSR2 records two adjacent $64 \mathrm{MHz}$ bands simultaneously at $20 \mathrm{~cm}$.

References.- (1) Sandhu et al. 1997; (2) Sandhu 2001; (3) van Straten 2003; (4) Hotan et al. 2006.
} 
TABLE 2

PSR J0437-4715 Timing Model Parameters ${ }^{\mathrm{a}}$

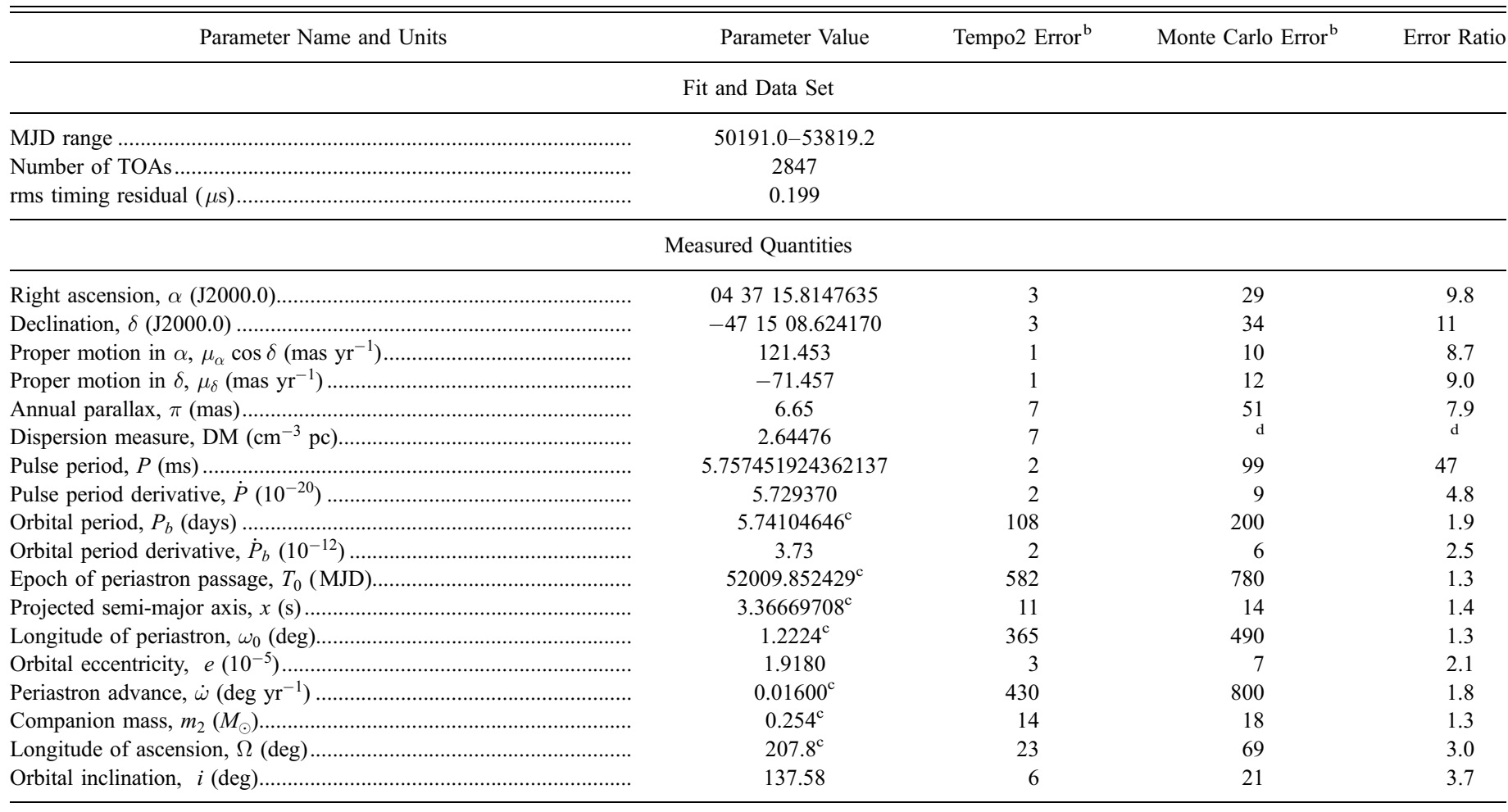

Set Quantities

Reference epoch for $P, \alpha$ and $\delta$ determination (MJD).... 52005

Reference epoch for DM determination (MJD) ............................... 53211

NOTE.-Units of right ascension are hours, minutes, and seconds, and units of declination are degrees, arcminutes, and arcseconds.

a These parameters are determined using Tempo2, which uses the International Celestial Reference System and Barycentric Coordinate Time. As a result this timing model must be modified before being used with an observing system that inputs Tempo format parameters. See Hobbs et al.(2006) for more information.

${ }^{\mathrm{b}}$ Given uncertainties are $1 \sigma$ values in the last digits of the parameter values.

${ }^{c}$ Because of large covariances, extra precision is given for selected parameters.

${ }^{\mathrm{d}}$ Dispersion measure was determined through alignment of simultaneous CPSR2 observations centered at $1341 \mathrm{MHz}$ and $1405 \mathrm{MHz}$. The effect of red noise is therefore not applicable.

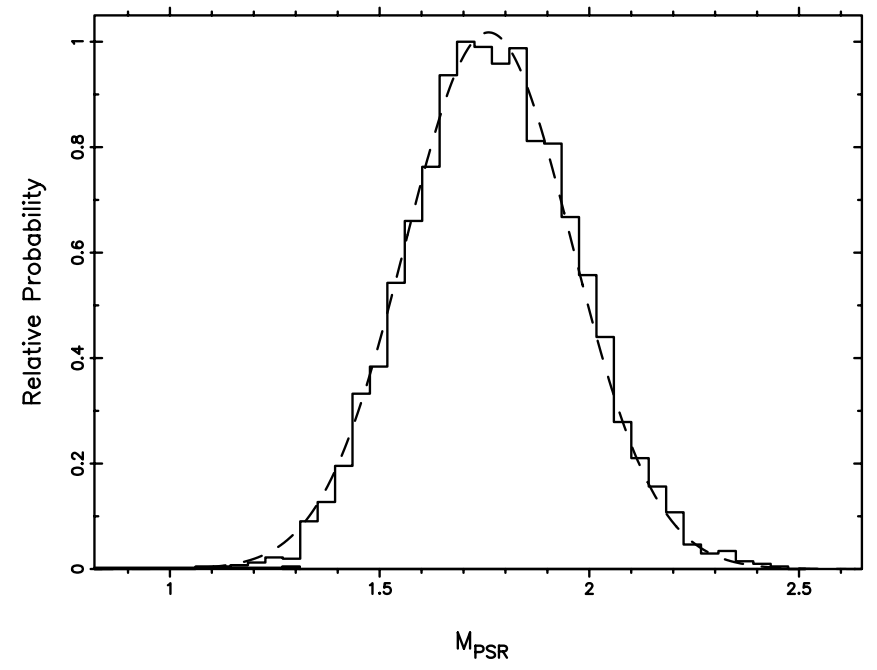

FIG. 2.-Pulsar mass probability distribution. The solid line shows the histogram of 5000 pulsar masses derived from a Monte Carlo simulation with power spectrum and sampling equal to that of the PSR J0437-4715 data set. The dashed line is a Gaussian distribution with a mean value of $m_{\mathrm{psr}}=1.76 M_{\odot}$ and standard deviation of $0.20 M_{\odot}$. expect the spectrum of these most precise data points to contain less low-frequency noise than the 10 year data set as a whole. We therefore expect the errors resulting from this analysis to be slightly overestimated. Ongoing research into extending the fitting routine with reliable whitening schemes to avoid the spectral leakage and hence improve the reliability of the measured parameters, is expected to reduce these errors by factors of around 2 . All errors given in this paper are those resulting from the Monte Carlo simulations, unless otherwise stated. The simulations also showed that any biases resulting from the red noise are statistically negligible for the reported parameters. (A full description of this Monte Carlo technique and the whitening schemes mentioned will be detailed in a future publication.)

\subsection{Solar System Ephemerides}

Pulsar timing results are dependent on accurate ephemerides for the solar system bodies. The results presented in this paper were obtained using the DE405 model (Standish 2004) and, for comparison, selected parameters obtained with the earlier DE200 model are shown in Table 3 . The greatly reduced $\chi^{2}$ indicates that the newer solar system ephemerides are superior to the earlier DE200, reinforcing similar conclusions of other authors (Splaver et al. 2005; Hotan et al. 2006). We notice the parallax value changes by more than $10 \sigma$, and that the different derived values 
TABLE 3

Comparison of DE200 and DE405 Results for PSR J0437-4715

\begin{tabular}{|c|c|c|}
\hline Parameter Name & DE200 Result & DE405 Result \\
\hline Rms residual (ns) & 281 & 199 \\
\hline Relative $\chi^{2}$ & 2.01 & 1.0 \\
\hline Parallax, $\pi$ (mas) & 7.84(7) & $6.65(7)$ \\
\hline Parallax distance, $D_{\pi}(\mathrm{pc})$ & $127.6(11)$ & $150.4(16)$ \\
\hline Previously published $\pi$ (mas) & $7.19(14)^{\mathrm{a}}$ & $6.3(2)^{\mathrm{b}}$ \\
\hline Kinematic distance, $D_{k}(\mathrm{pc})$ & $154.5(10)$ & $156.0(10)$ \\
\hline$D_{k}$ corrected for Galactic effects $(\mathrm{pc})$ & $155.5(10)$ & $157.0(10)$ \\
\hline Variation of Newton's gravitational constant, $|\dot{G} / G|\left(10^{-12} \mathrm{yr}^{-1}\right)$ & $-21.2(22)^{\mathrm{c}}$ & $-5.0(26)^{\mathrm{c}}$ \\
\hline 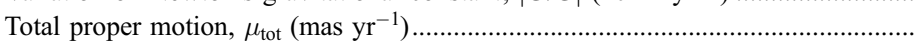 & $140.852(1)$ & $140.915(1)$ \\
\hline Companion mass, $m_{c}\left(M_{\odot}\right)$ & $0.263(14)$ & $0.254(14)$ \\
\hline Pulsar mass, $m_{\mathrm{psr}}\left(M_{\odot}\right)$ & $1.85(15)$ & $1.76(15)$ \\
\hline Periastron advance, $\dot{\omega}\left(\operatorname{deg} \mathrm{yr}^{-1}\right)$ & $0.020(4)$ & $0.016(4)$ \\
\hline GR prediction of $\dot{\omega}\left(\operatorname{deg} \mathrm{yr}^{-1}\right)$ & $0.0178(9)$ & $0.0172(9)$ \\
\hline
\end{tabular}

NoтE.-Numbers in parentheses represent the formal Tempo2 $1 \sigma$ uncertainty in the last digits quoted, unless otherwise stated.

a From van Straten (2001).

b From Hotan et al. (2006).

${ }^{c}$ Given are $2 \sigma$ errors, i.e., $95 \%$ confidence levels.

are closely correlated with the ephemeris used. Although the effect is not as dramatic as it appears because of the underestimation of the Tempo 2 errors, the fact that the DE405 results agree much better with the more accurate kinematic distance (discussed in the next section), strongly suggests that the differences are due to the ephemeris used and confirms that the DE405 ephemeris is superior. Finally, we note that the DE405 measurement of $\dot{\omega}$ $\left(0.016 \pm 0.008 \mathrm{deg} \mathrm{yr}^{-1}\right)$ is consistent with the GR prediction

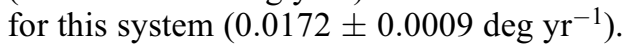

\section{KINEMATIC DISTANCE}

As shown in Figure 3, the long-term timing history enables precise measurement of the orbital period derivative, $\dot{P}_{b}=(3.73 \pm$ $0.06) \times 10^{-12}$. This observed value represents a combination of phenomena that are intrinsic to the binary system and dynam-

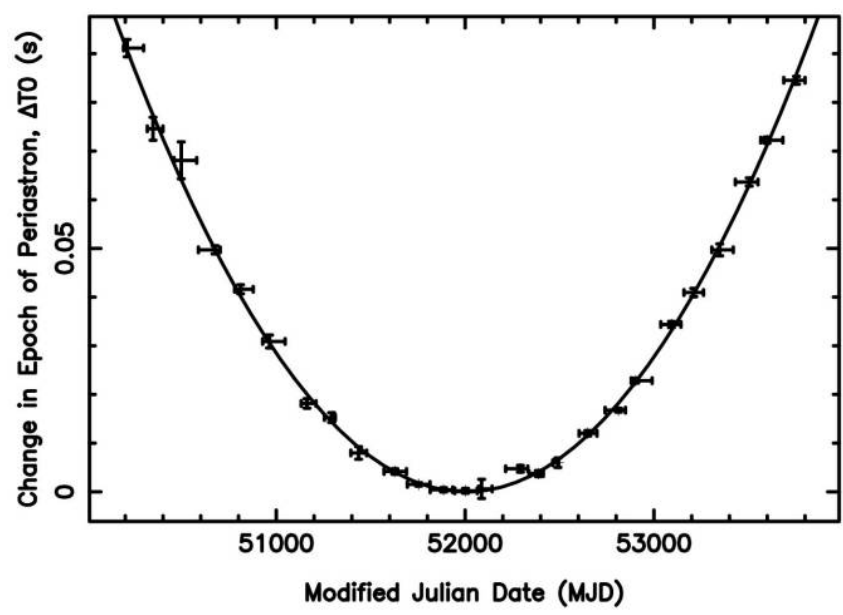

FIG. 3.-Variations in epoch of periastron passage $\left(T_{0}\right)$ due to apparent orbital period increase. A steady increase in orbital period is equivalent to a quadratic increase in $T_{0}$ relative to periastron times for a constant orbital period. For this plot, $T_{0}$ was measured on data spans of up to 120 days with a model having no orbital period derivative. The formal $1 \sigma$ measurement errors reported by Tempo 2 are shown by vertical error bars and the epochs over which the measurements were made are shown by horizontal bars. As the mean measurement time was determined through a weighted average of the data contained in the fit, these horizontal bars need not be centered at the mid time associated with the measurement. The parabola shows the effect of the $\dot{P}_{b}$ value obtained from a fit to the data shown in Fig. 1 . ical effects that result in both real and apparent accelerations of the binary system along the line of sight (Bell \& Bailes 1996); i.e.

$$
\dot{P}_{b}^{\text {obs }}=\dot{P}_{b}^{\text {int }}+\dot{P}_{b}^{\text {Gal }}+\dot{P}_{b}^{\text {kin }},
$$

where "obs" and "int" refer to the observed and intrinsic values; "Gal" and "kin" are the Galactic and kinematic contributions.

Intrinsic orbital decay is a result of energy loss typically due to effects such as atmospheric drag and tidal dissipation; however, in a neutron star-white dwarf binary system like PSR J0437-4715, energy loss is dominated by quadrupolar gravitational wave emission. For this system, GR predicts (Taylor \& Weisberg 1982) $\dot{P}_{b}^{\mathrm{GR}}=-4.2 \times 10^{-16}, 2$ orders of magnitude smaller than the uncertainty in the measured value of $\dot{P}_{b}$.

Galactic contributions to the observed orbital period derivative include differential rotation and gravitational acceleration (Damour \& Taylor 1991). The differential rotation in the plane of the Galaxy is estimated from the Galactic longitude of the pulsar and the Galactocentric distance and circular velocity of the Sun. Acceleration in the Galactic gravitational potential varies as a function of height above the Galactic plane (Holmberg \& Flynn 2004), which may be estimated using the parallax distance and the Galactic latitude of the pulsar. Combining these terms gives $\dot{P}_{b}^{\text {Gal }}=(-1.8-0.5) \times 10^{-14}=-2.3 \times 10^{-14}$, which is of the same order as the current measurement error.

Given the negligible intrinsic contribution, equation (1) can be simplified and rewritten in terms of the dominant kinematic contribution known as the Shklovskii effect (Shklovskii 1970), an apparent acceleration resulting from the nonlinear increase in radial distance as the pulsar moves across the plane perpendicular to the line of sight; quantified by the proper motion, $\mu$, and distance $D$ from the Earth:

$$
\dot{P}_{b}^{\text {obs }}-\dot{P}_{b}^{\text {Gal }} \simeq \dot{P}_{b}^{\text {kin }}=\frac{\mu^{2} D}{c} P_{b},
$$

where $c$ is the vacuum speed of light. Using the measured values of $\mu, P_{b}$, and $\dot{P}_{b}$, equation (2) is used to derive the kinematic distance (Bell \& Bailes 1996): $D_{k}=157.0 \pm 2.4$ pc. This distance is consistent with the one derived from parallax $\left(D_{\pi}=\right.$ $150 \pm 12 \mathrm{pc}$; see also Fig. 4) and is, with a relative error of $1.5 \%$, comparable in precision to the best parallax measurements from VLBI (Torres et al. 2007) and better than typical 


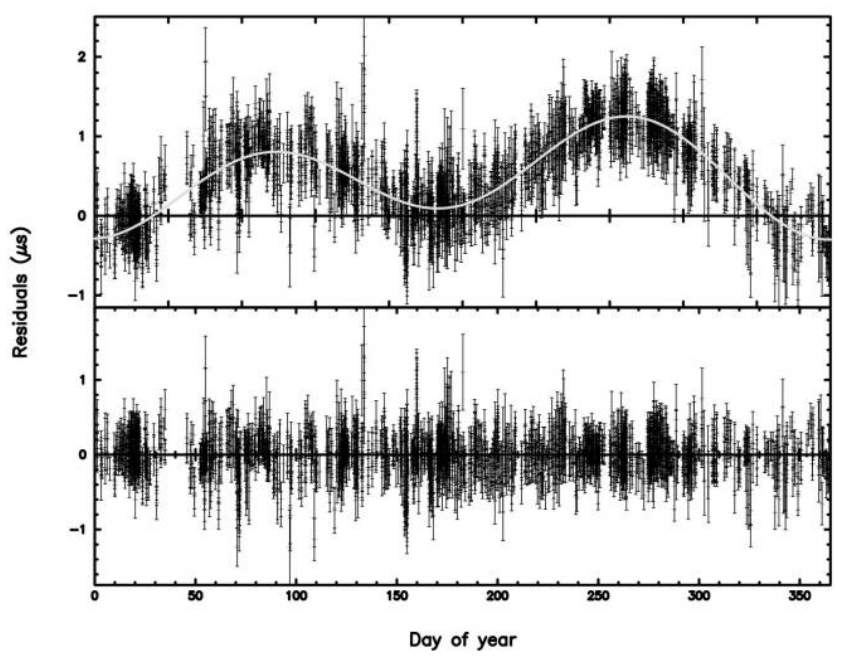

FIG. 4.-Parallax signature of PSR J0437-4715. Top: Timing residuals for PSR J0437-4715 as a function of day of year (starting on 18 November), without parallax but with all remaining parameters at their best-fit values. The smooth curve represents the model fit of a parallax of 6.65 mas. Bottom: The same timing residuals with parallax included in the model. The overall $\mathrm{rms}$ for the top and bottom plots is 524 and $199 \mathrm{~ns}$, respectively. The double-humped signature specific to parallax originates from the delay in pulse time of arrival (TOA) as the Earth orbits the Sun and samples different parts of the curved wave front originating at the pulsar.

relative errors provided by the Hipparcos and Hubble space telescopes (Valls-Gabaud 2007).

Given the dependence of parallax distances on ephemerides, as described in $\S 2.3$, it is interesting to note the robustness of $D_{k}$. Also, Table 2 shows that the presence of red noise corrupts the parallax error by a factor of 7.9 , whereas $\dot{P}_{b}$ is only affected by a factor of 2.5. These facts clearly indicate the higher reliability of $D_{k}$ as compared to $D_{\pi}$.

\section{LIMITS ON $\dot{P}_{b}$ ANOMALIES: $\dot{G}$ AND}

THE ACCELERATION OF THE SOLAR SYSTEM

Any anomalous orbital period derivative can be constrained by substituting the parallax distance into equation (2), yielding

$\left(\frac{\dot{P}_{b}}{P_{b}}\right)^{\text {excess }}=\left(\dot{P}_{b}^{\text {obs }}-\dot{P}_{b}^{\mathrm{Gal}}-\dot{P}_{b}^{\mathrm{kin}}\right) / P_{b}=(3.2 \pm 5.7) \times 10^{-19} \mathrm{~s}^{-1}$.

in which the error is almost exclusively due to the parallax uncertainty. Following Damour \& Taylor (1991) this can be translated into a limit on the time derivative of Newton's gravitational constant (given are $95 \%$ confidence levels):

$$
\frac{\dot{G}}{G}=-\frac{1}{2}\left(\frac{\dot{P}_{b}}{P_{b}}\right)^{\text {excess }}=(-5 \pm 18) \times 10^{-12} \mathrm{yr}^{-1}
$$

This limit is of the same order as those previously derived from pulsar timing (see $\S 1$ ), but a currently ongoing VLBI campaign on this pulsar is expected to improve significantly on our parallax measurement, and this should improve our limit to close to that put by LLR $\left[(4 \pm 9) \times 10^{-13} \mathrm{yr}^{-1}\right.$; Williams et al. 2004] The LLR experiment is based on a complex $n$-body relativistic model of the planets that incorporates over 140 estimated parameters, such as elastic deformation, rotational dissipation and two tidal dissipation parameters. In contrast, the PSR J0437-4715 timing result is dependent on a different set of models and as- sumptions, and therefore provides a useful independent confirmation of the LLR result.

A recent investigation into the possible causes of a measured variability of the astronomical unit (AU; Krasinsky \& Brumberg 2004) has refuted all but two sources of the measured value of $d \mathrm{AU} / d t=0.15 \pm 0.04 \mathrm{~m} / \mathrm{yr}$. Krasinsky \& Brumberg (2004) state that the measured linear increase in the AU would be due to either systematic effects or to a time variation of $G$ at the level of $\dot{G} / G=(-10 \pm 3) \times 10^{-13} \mathrm{yr}^{-1}$, comparable to, but inconsistent with, the LLR limit.

The anomalous $\dot{P}_{b}$ measurements of a number of millisecond pulsars have also been used to place limits on the acceleration of the solar system due to any nearby stars or undetected massive planets (Zakamska \& Tremaine 2005). The PSR J0437-4715 data set limits any anomalous solar system acceleration to $\left|a_{\odot} / c\right| \leq 1.5 \times 10^{-18} \mathrm{~s}^{-1}$ in the direction of the pulsar with $95 \%$ certainty. This rules out any Jupiter-mass planets at distances less than $117 \mathrm{AU}$ along the line of sight, corresponding to orbital periods of up to $1270 \mathrm{yr}$. Similarly, this analysis excludes any Jupiter-mass planets orbiting PSR J0437-4715 between $\sim 5$ and $117 \mathrm{AU}$ along the line of sight. Zakamska \& Tremaine (2005) also compared the sensitivity of this limit to that of optical and infrared searches for trans-Neptunian objects (TNOs) and concluded that beyond $\sim 300$ AU the acceleration limit becomes more sensitive than the alternative searches. At a distance of $300 \mathrm{AU}$ from the Sun, the $95 \%$ confidence upper limit on the mass of a possible TNO (in the direction of the pulsar) is 6.8 Jupiter masses. The precise VLBI measurement of parallax mentioned above might decrease this to close to one Jupiter mass.

\section{PULSAR MASS}

A combination of the mass function and a measurement of the Shapiro delay range can be used to obtain a measurement of the pulsar mass. Using this method, van Straten et al. (2001) derived a mass for PSR J0437-4715 of $1.58 \pm 0.18 M_{\odot}$, whereas Hotan et al. (2006) obtained $1.3 \pm 0.2 M_{\odot}$. It should be noted, however, that these values resulted from a model that incorporated geometric parameters first described by Kopeikin $(1995,1996)$, but covariances between these and other timing parameters (most importantly the companion mass or Shapiro delay range) were not taken into account. While the length of the data sets used by these authors were only a few years, it can also be expected that some spectral leakage from low-frequency noise was unaccounted in the errors of these previously published values. As described in $\S 2$, the Monte Carlo simulations and extended fitting routines implemented for the results reported in this paper do include these covariances and spectral leakage; it can therefore be claimed that the current estimates (at 68\% confidence) of $m_{c}=0.254 \pm 0.018 M_{\odot}$ and $m_{\mathrm{psr}}=1.76 \pm 0.20 M_{\odot}$, for the white dwarf companion and pulsar respectively, reflect the measurement uncertainty more realistically than any previous estimate. The distribution of $m_{\mathrm{psr}}$ that follows from the 5000 Monte Carlo realizations is shown in Figure 2, together with a Gaussian with mean 1.76 and standard deviation 0.20 . This demonstrates the symmetric distribution of the pulsar mass likelihood distribution, induced by the precise determination of the orbital inclination angle.

We also note that the new mass measurement of PSR J04374715 is the highest obtained for any pulsar to date. Distinction needs to be made between the objective mass estimate presented in this paper and the subjective mass predictions presented in Ransom et al. (2005) and Freire et al. (2008). The pulsar mass confidence interval presented in this paper is derived from the measurement uncertainties of all relevant model parameters, 
including the well-determined orbital inclination angle, $i$. In contrast, $i$ is unknown in the Terzan $5 \mathrm{I}$ and $\mathrm{J}$ (Ransom et al. 2005) and PSR J1748-2021B (Freire et al. 2008) binary systems, and the posterior probability intervals for the pulsar masses presented in these works are based on the prior assumption of a uniform distribution of $\cos i$. These fundamental differences must be accounted for in any subsequent hypothesis testing. Consequently, PSR J0437-4715 is currently the only pulsar to provide reliable constraints on equations of state based on hyperons and Bose-Einstein condensates as described by Lattimer \& Prakash (2007). Simulations with Tempo2 indicate that a forthcoming observational campaign with a new generation of backend systems can be expected to increase the significance of this measurement by another factor of about 2 in the next year.

\section{CONCLUSIONS}

We have presented results from the highest precision longterm timing campaign to date. With an overall residual rms of $199 \mathrm{~ns}$, the $10 \mathrm{yr}$ of timing data on PSR J0437-4715 have provided a precise measurement of the orbital period derivative, $\dot{P}_{b}$, leading to the first accurate kinematic distance to a millisecond pulsar: $D_{k}=157.0 \pm 2.4$ pc. Application of this method to other pulsars in the future can be expected to improve distance estimates to other binary pulsar systems (Bell \& Bailes 1996).

Another analysis based on the $\dot{P}_{b}$ measurement places a limit on the temporal variation of Newton's gravitational constant. We find a bound comparable to the best so far derived from pulsar timing: $\dot{G} / G=(-5 \pm 18) \times 10^{-12} \mathrm{yr}^{-1}$. An ongoing VLBI campaign on this pulsar is expected to improve this limit, enabling an independent confirmation of the LLR limit.

Previous estimates of the mass of PSR J0437-4715 have been revised upwards to $m_{\mathrm{psr}}=1.76 \pm 0.20 M_{\odot}$, which now makes it one of the few pulsars with such a heavy mass measurement. A new generation of back-end instruments, dedicated observing campaigns, and data prewhitening techniques currently under development should decrease the error in this measurement enough to significantly rule out various equations of state for dense nuclear matter.

The Parkes Observatory is part of the Australia Telescope which is funded by the Commonwealth of Australia for operation as a National Facility managed by CSIRO. We thank the staff at Parkes Observatory for technical assistance during regular observations. B. A. J holds a National Research Council Research Associateship Award at the Naval Research Laboratory (NRL). Basic research in radio astronomy at NRL is supported by the Office of Naval Research. The authors wish to express their gratitude to William Coles from the University of California at San Diego, for extensive discussion and help with the Monte Carlo error analysis. Finally, we thank the referee for valuable and inspiring comments.

Facilities: Parkes
Becker, W., \& Trümper, J. 1993, Nature, 365, 528

Bell, J. F., \& Bailes, M. 1996, ApJ, 456, L33

Bell, J. F., Bailes, M., \& Bessell, M. S. 1993, Nature, 364, 603

Britton, M. C. 2000, ApJ, 532, 1240

Damour, T., \& Deruelle, N. 1986, Ann. Inst. H. Poincaré, 44, 263

Damour, T., \& Taylor, J. H. 1991, ApJ, 366, 501

Edwards, R. T., Hobbs, G. B., \& Manchester, R. N. 2006, MNRAS, 372,1549

Freire, P. C. C., Ransom, S. M., Begin, S., Stairs, I. H., Hessels, J. W. T., Frey, L. H., \& Camilo, F. 2008, 675, 670

Hobbs, G. B., Edwards, R. T., \& Manchester, R. N. 2006, MNRAS, 369, 655

Holmberg, J., \& Flynn, C. 2004, MNRAS, 352, 440

Hotan, A. W., Bailes, M., \& Ord, S. M. 2006, MNRAS, 369, 1502

Hotan, A. W., van Straten, W., \& Manchester, R. N. 2004, Proc. Astron. Soc. Australia, 21, 302

Johnston, S., et al. 1993, Nature, 361, 613

Kaspi, V. M., Taylor, J. H., \& Ryba, M. 1994, ApJ, 428, 713

Kopeikin, S. M. 1995, ApJ, 439, L5 . 1996, ApJ, 467, L93

Krasinsky, G. A., \& Brumberg, V. A. 2004, Celest. Mech. Dyn. Astron., 90, 267

Lattimer, J. M., \& Prakash, M. 2007, Phys. Rep., 442, 109

Nordtvedt, K. 1990, Phys. Rev. Lett., 65, 953

Ransom, S. M., Hessels, J. W. T., Stairs, I. H., Freire, P. C. C., Camilo, F., Kaspi, V. M., \& Kaplan, D. L. 2005, Science, 307, 892

Sandhu, J. S. 2001. Ph.D. thesis, Caltech
REFERENCES

Sandhu, J. S., Bailes, M., Manchester, R. N., Navarro, J., Kulkarni, S. R., \& Anderson, S. B. 1997, ApJ, 478, L95

Shklovskii, I. S. 1970, Soviet Astron.-AJ, 13, 562

Splaver, E. M., Nice, D. J., Stairs, I. H., Lommen, A. N., \& Backer, D. C. 2005, ApJ, 620, 405

Standish, E. M. 2004, A\&A, 417, 1165

Staveley-Smith, L., et al. 1996, Publ. Astron. Soc. Australia, 13, 243

Taylor, J. H. 1993, in Particle Astrophysics, ed. G. Fontaine \& J. Tran Thanh van (Gif-sur-Yvette: Editions Frontières), 367

Taylor, J. H., \& Weisberg, J. M. 1982, ApJ, 253, 908

Torres, R. M., Loinard, L., Mioduszewski, A. J., \& Rodriguez, L. F. 2007, ApJ, 671,1813

Valls-Gabaud, D. 2007, in IAU Symp. 240, Binary Stars as Critical Tools \& Tests in Contemporary Astrophysics, ed. W. I. Hartkopf, E. F. Guinan, \& P. Harmanec (Cambridge: Cambridge Univ. Press), 281

van Straten, W. 2003. Ph.D. thesis, Swinburne Univ. of Technology

. 2004, ApJS, 152, 129 2006, ApJ, 642, 1004

van Straten, W., Bailes, M., Britton, M., Kulkarni, S. R., Anderson, S. B., Manchester, R. N., \& Sarkissian, J. 2001, Nature, 412, 158

Williams, J. G., Turyshev, S. G., \& Boggs, D. H. 2004, Phys. Rev. Lett., 93, 261101

You, X.-P., et al. 2007, MNRAS, 378, 493

Zakamska, N. L., \& Tremaine, S. 2005, AJ, 130, 1939 